\title{
Engagement and Retention in HIV Care for Transgender Women: Perspectives of Medical and Social Service Providers in New York City
}

\author{
Walter Bockting, PhD, Caitlin MacCrate, BA, Hayley Israel, MD, \\ Joanne E. Mantell, PhD, and Robert H. Remien, PhD
}

\begin{abstract}
Transgender women are less likely to engage in HIV care and adhere to antiretroviral medications than other atrisk populations. Health care and social service providers, in addition to consumers, have experiences that can elucidate barriers and facilitators to care and inform interventions. Guided by the social/ecological model, we conducted interviews with 19 providers working with transgender women. At the health systems level, perceived barriers included lack of care accessibility and security, providers' misunderstanding of the transgender community, and lack of cultural competency of information systems and staff. At the community level, barriers included HIV stigma. At the family level, barriers included rejection and housing instability. At the individual level, barriers included conflicts between HIV- and transgender care, medication side effects, competing priorities, mental health issues and substance abuse, and low health literacy. Facilitators included provider competence in transgender health, improved access to care, and patient empowerment. Findings indicate the need for an integrated care model.
\end{abstract}

Keywords: access to care, transgender, retention in care, engagement in care

\section{Introduction}

$\mathbf{O}$ PTIMIZING ENGAGEMENT AND retention in HIV care for all people living with HIV is crucial for ending the HIV/AIDS epidemic in the United States. As of December 2015, New York State had the highest reported number of people diagnosed with HIV/AIDS in the United States with a total of 132,280 cases $;{ }^{1} 22,945$ of these cases were in New York. ${ }^{2}$ Retention of HIV-positive persons in health care with suppression of viral load is central to the goal of decreasing the burden of HIV/AIDS. ${ }^{3,4}$ Adherence to antiretroviral treatment (ART) and suppression of viral load lead to reduced morbidity and mortality for people living with HIV, decreased HIV transmission within the population, ${ }^{5-9}$ and improved quality of life. ${ }^{10}$

The health care needs of highly vulnerable populations living with HIV, such as transgender women, must be considered in designing strategies for improving engagement and retention in care. Transgender women living with HIV are a population with a significant HIV burden in both the United States and worldwide. ${ }^{11-14}$ A 2007 meta-analysis indicated that as many as $27.7 \%$ of transgender women in the United States tested positive for HIV, with up to $48 \%$ engaging in risky sexual behaviors such as unprotected anal intercourse and sex work. ${ }^{11}$ A 2013 meta-analysis indicated a disproportionate burden of HIV infection globally among transgender women: a $19.1 \%$ HIV prevalence and an odds ratio of 48.1 for seropositivity compared with all other adults of reproductive age. ${ }^{13}$

Studies have also shown that transgender women are less likely to be tested for HIV than other gender and sexual minorities [transgender men, men who have sex with men (MSM)], less likely to initiate ART than other people living with HIV, and less likely to adhere to ART. ${ }^{14,15}$ Analysis of the iPrEX Trial data showed that among study participants on daily pre-exposure prophylaxis (PrEP), transgender women were less adherent than MSM. ${ }^{16}$ These findings suggest there are unique barriers to adherence to ART for transgender women living with HIV. 
Recognition that transgender women are at high risk for HIV has led to the development of only a few transgenderspecific HIV prevention strategies that show promise in terms of their feasibility and acceptability, as well as their effectiveness. These strategies include cultural competence training, behavioral interventions for reducing HIV risk behaviors (unsafe sex or injection practices), and HIV prevention within the context of promoting overall transgender health. ${ }^{17-20}$

Less is known, however, about how to improve engagement and retention in HIV care among transgender women living with HIV. A few qualitative studies have addressed the perspectives of transgender women living with HIV. ${ }^{21,22}$ These studies highlight the multiple barriers that transgender women face in engaging in HIV care, such as negative experiences with providers who were uneducated or insensitive to transgender issues, the burden of multiple stigmatized identities, and the prioritization of survival (i.e., basic needs such as housing) and gender-affirming, transition-related care (including hormone therapy) over HIV care. However, an important but understudied area in the care of transgender women living with HIV is the perspective of health care and social service providers, who have experiences from the provider's point of view interacting with the health care system as well as with the target population.

To address this gap, the current study explored providers' perceptions of barriers and facilitators to engagement in HIV care among transgender women, their insights about structural and patient characteristics that may influence engagement in care, and their suggestions for improvement. Health and social service providers working with transgender women living with HIV offer valuable insights that may be incorporated in the development of tailored interventions to improve engagement and retention in care.

\section{Methods}

\section{Participants and procedures}

The study was approved by the Institutional Review Board of the New York State Psychiatric Institute/Columbia Psychiatry. Participants were eligible for study interviews if they were 18 years old or older, English speaking, and a medical or social service provider knowledgeable about transgender women living with HIV in New York City. We used convenience sampling and recruited participants through health and social service organizations known to our HIV Center as serving a critical mass of transgender women; all of these organizations were engaged, and the vast majority are represented in the sample. We also accepted referrals from participants already interviewed. Organizations were of medium-to-large size, included Federally Qualified Health Centers, and offered medical and/or social service programs. We completed data collection when saturation was achieved and no new important insights from providers emerged.

We constructed an interview guide based on the social/ ecological model $\mathrm{l}^{23-25}$ to explore barriers and facilitators to care on multiple levels, including health and social service systems, community, family, and the individual level (Appendix A). The goal was to identify potential points for intervention in real-world settings that provide HIV care or facilitate and support engagement and retention in care. Interviews were conducted by the first and fourth author of this article, who kept detailed field notes. Interviews were audiorecorded and subsequently transcribed verbatim. Interviews lasted $\sim 90$ min each. Data collection was completed in 2014.

\section{Data analysis}

A conventional content analysis was performed. ${ }^{26}$ Two analysts read all transcripts and independently coded a subset of the interview transcripts manually, developing codes based on each level of the social/ecological model (i.e., health systems, community, family, individual) to identify themes related to barriers and facilitators to engagement and retention in HIV care. The analysts met repeatedly to review codes and to discuss and resolve discrepancies. For example, "Systems barriers" was a primary coding category, and within this category, there were subthemes of "lack of services available in the evening/night," "lack of services in safe/accessible areas," and "mistrust of doctors." Analysts then coded the entire data set; interrater reliability was $80 \%$ (manually computed agreement between the two coders).

Results were interpreted and integrated through review and discussion among members of the study's interdisciplinary research team, which included experts in clinical and educational psychology, social work, sociology, medicine, and public health.

\section{Results}

\section{Description of the sample}

Sixteen interviews were conducted with 19 participants; 3 interviews were conducted simultaneously with 2 participants who worked at the same organization. Eight participants identified as cisgender women, five as transgender women, and six as cisgender men. The fact that participants included transgender-identified providers meant that their perspectives, at times, included their own experiences as members of the community being served. Participants were diverse in race/ethnicity. Eight participants identified as nonHispanic white, seven as black, two as Hispanic, and two as Asian. Six participants were medical providers and 13 were social service providers, representing a total of 14 organizations in the New York City metropolitan area.

\section{Barriers by level of the social/ecological model}

Guided by our theoretical framework, we present insights from participants on barriers and facilitators to engagement and retention in HIV care organized by level of the social/ecological model: Health systems, community, family, and individual (Fig. 1).

Health and social service system-level barriers. Themes related to health and social service system-level barriers included concerns about transgender sensitivity, accessibility, convenience of hours, confidentiality, and health care administration.

Transgender sensitivity. Participants reported that HIV care services often did not consider transgender women's identities and experiences, making transgender women feel unwelcome. For example, clinic forms typically did not allow transgender patients to indicate their gender identities correctly or misclassified them as MSM. Participants reported that many 


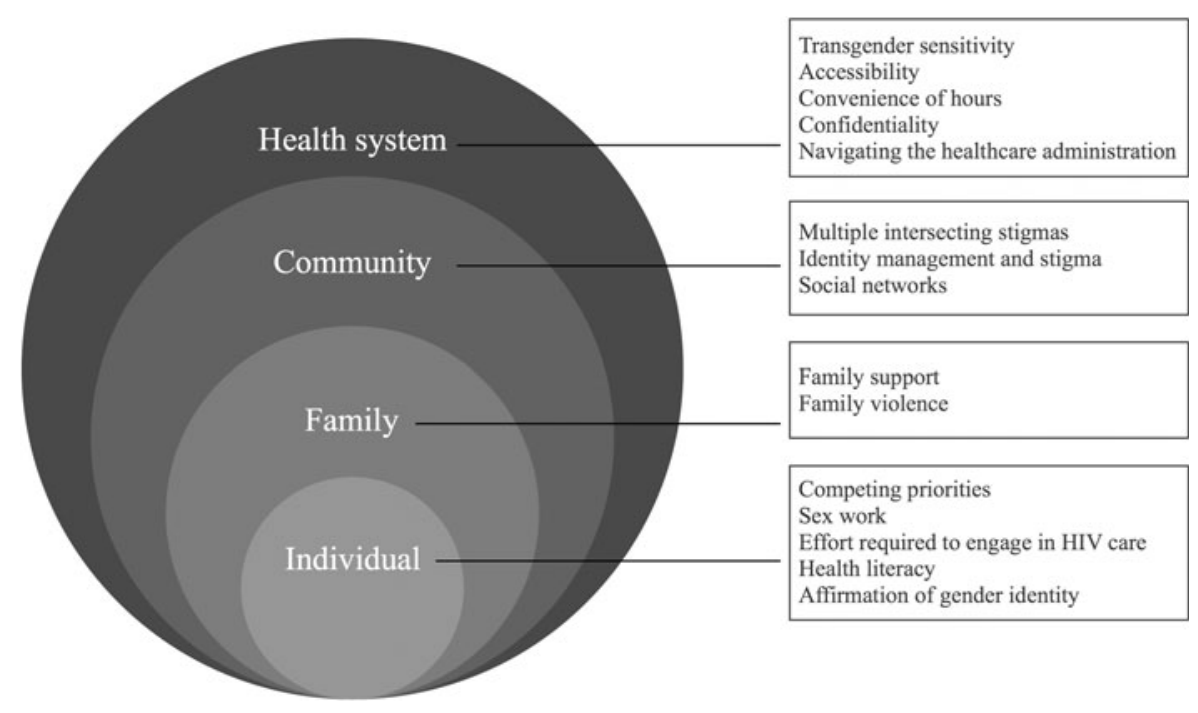

FIG. 1. Barriers and facilitators to engagement and retention in HIV care by level of the social/ecological model. providers are uncomfortable providing care to transgender women due to a lack of training or preparation.

Many providers did not know how to manage genderaffirming medical interventions such as hormone therapy and surgery, much less coordinate gender-affirming care with HIV care. Some participants had witnessed unprofessional behavior and outright violations of privacy by providers related to a lack of transgender sensitivity, such as inappropriate disclosure of a patient's transgender status. Experiences of bias and stigma related to a patient's gender identity and expression were viewed as contributing to a general feeling of mistrust of the medical profession among transgender women.

As one participant, who identified as a transgender woman, stated: “... [my] psychologist did not believe in transsexuality, so he attempted to get me to revert, saying that I should just dress in drag or be a cross-dresser because this is really just something that I imagined in my mind." Another participant recounted a patient's interactions with hospital staff who were insensitive to her gender expression:

[She] had a terrible health situation and ended up going to [the hospital] ... and she wasn't there [for more than] five or ten minutes [when] the buzz on the floor was, 'Oh, my God, she's got a penis' or, 'Oh, my God, he's got breasts.' And they declined to call her by her name, but called her by what it says on her I.D. card. And they were magnificently inappropriate with her ... such that a few people got together and picketed outside the place and called in [name] who talked to the hospital administrator before we got it straightened out.

A lack of sensitivity was seen as having far-reaching consequences at times:

One of, um, you know, one of the girls I knew, I got her into housing because we went through the health department and all that stuff and HASA [HIV/AIDS Services Administration, an organization which provides case management and assistance in applying for public benefits and services], and when she got into housing, she let it slip away because she went to the office in the morning and no-one would help her or they called her by her male name, you know. There [are] so many things that cause these girls not to go into treatment.

Another participant noted that a transgender woman had fled safety-net housing segregated by sex. She was forced to live on the men's floor after struggling to fit in on the women's floor:
If you were a trans, uh, male-to-female, um, so she was down on the girls' floor. She was fighting a lot, and then they decided to put her on the boys' floor and she refused to do that because she didn't want to be on the boys' floor and she left and went back to that apartment. So she didn't even stay there long enough to access any of [the medical or social services].

Accessibility. Participants shared that, aside from a lack of transgender sensitivity and inclusion, transgender women living with HIV faced barriers to care related to accessibility and availability more generally. To access care, transgender women need to reach venues where services are being offered and feel safe in doing so. Participants in our study discussed at length their concerns about clinic locations, both in terms of physical safety (potentially requiring an escort for protection) and socioeconomic status (feelings of not fitting in), including at clinics targeting the Lesbian, Gay, Bisexual, and Transgender (LGBT) community:

When trying to link them to, like, let's say the [name] program, the fact that it's out in [location] and that's not an environment a trans girl feels comfortable just walking around. Um, it's a problem. You know, there's few places where they feel comfortable or can be ... you know, one of the things we do here is we escort people to care.

\section{And:}

Participant: The girls do not feel comfortable going to [a specific LGBT clinic].

Interviewer: They don't? And why is that?

Participant: Um, they feel like they're looked down upon. Again, they feel like, you know, they don't make the right amount of money, they don't live in Chelsea, they don't have the Hell's Kitchen attitude so people are going to ... you know, it's like they ... they come in already expecting to be put down so anything that happens they're gonna ... a receptionist doesn't smile at them, they're gonna think...

Convenience of hours. Participants noted the lack of services in the evening or nighttime as a barrier to care for transgender women living with HIV. Reasons given why transgender women need access during these hours include reluctance on the part of some women to go out during the 
day due to fear of not being able to pass as a woman and/or the need to sleep during the day after engaging in sex work late at night:

...scheduling, um, appointments later in the day would be most suited for these kinds of groups because again, some of these girls, they're not up until 4:00 or 5:00 in the afternoon.

Confidentiality. Participants noted that many transgender women, like other persons living with HIV, feel uncomfortable accessing care at a location known for specifically providing HIV care. Simply entering the doors of these spaces can be seen as revealing one's HIV status. Similarly, participants reported that many transgender women were hesitant to seek HIV care at locations specifically providing care to the LGBT community, because of the risk of unintended disclosure of one's HIV status in a community as small as the transgender community. One participant suggested that if a clinic were located in the heart of a neighborhood where many transgender women live, they would opt to go elsewhere for a higher level of discretion to prevent gossip about seeking HIV care services:

It's like you go to the doctor and you see someone at the doctor, it's ... it boggles me and here they come, "Oh, you know such and such was at the doctor. I know she has this, I know she has that." And if a clinic says that it is HIV-specific or we have HIV care on this day or we have an HIV group ... girls are not gonna want to go to it because they feel like their business is going to be put out on the street.

Navigating the health care system. Participants also mentioned barriers related to bureaucracy of the health care industry, such as inefficient procedures and poorly designed processes for accessing care. Any aspect of bureaucracy that delayed a patient's ability to receive treatment was viewed as an obstacle. For example, one participant noted that patients were frustrated by the multiple steps required to access gender-affirming hormone therapy at a certain clinic, leading to skepticism about accessing other services, such as HIV care:

Participant: [At clinic name], which a lot of people go to, has this, um, like it's the kind of go-to for LGBT ... but there's, like, barriers from the doctors. Like, you have to ... for hormone use they ... you have to be over 18 or you have to wait a certain ... have a certain amount of counseling sessions and I think because they're angry about that, it carries over into HIV stuff. They don't trust them with those kinds of things and so they're certainly not going to trust them with things that aren't even, um, tangible. Like, necessary right now.

Interviewer: Mm-hmm.

Participant: Transition is necessary and they're keeping that from me. Why would I go talk to them about this?" You know? Like, I get that feeling...

Thus, processes perceived as bureaucratic impeded participants from engaging in care, which may reflect a disconnect between the needs of some transgender women living with HIV and the needs of institutions, even those designed to meet the specific health care needs of transgender women.

Community-level barriers. Multiple intersecting stigmas. Transgender women as a group may face unique barriers to engagement and retention in care due to being a member of a stigmatized minority population. Nearly every participant in this study highlighted the impact of social stigma as a crucial hurdle that transgender women living with HIV face when deciding whether to seek care. Participants described stigma acting as a barrier to care within the general population directed at transgender women, as well as within the transgender community directed at transgender women and transgender sex workers living with HIV. Stigma then further affects the individual as it becomes internalized.

Transgender women living with HIV face the burden of having two highly stigmatized aspects of their identity (being transgender and HIV positive), and sometimes three or more (e.g., being transgender, HIV positive, engaged in sex work, and a person of color). Study participants described this intersection of multiple stigmatized identities often as overwhelming:

Participant: Trans women don't feel like they're gonna live long lives anyway because they're already extremely oppressed.

Interviewer: So $\ldots$ and the mindset changes once they are positive?

Participant: Once they become positive, it changes. They don't care; they continue to escort until the last minute or whenever they can. Some are on, um, Medical Assistance, but they don't take the hormones ... Condoms are optional ..." Interviewer: Tell us a little bit more about when you've had those conversations, what comes up in those talks with them? Participant: In those conversations, it's mind-boggling to me because these people... it's as though they have lost all, uh, how should I put it? They've lost the drive to live.

And:

The girls who had the ... how should I put this? The strength to walk out into society and say, "This is me, this is who I am, you're going to give me this job, or I'm not going to take it," are really, really, really prejudiced to those girls who are in sex work.

Identity management and stigma. Participants elaborated on gender identification and related internalized transphobia that may divide and isolate transgender women, making it less likely for some to feel comfortable seeking care. Some women reportedly are not comfortable with the label "transgender" or "trans" to describe their gender identity, and instead identify simply as women without a transgender adjective. According to our participants, this may lead them to avoid places that offer transgender-specific health care:

\footnotetext{
... not everybody is gonna want to go there, right? And, um, we see that because even ... I have a thing, I created something called a T-Lounge here. I asked this young girl ... I said, "You coming to the T-Lounge, girl?" She's like, "No." Because she doesn't want ... there's such a stigma of what trans means. She just wants to be a girl.
}

In New York, a segment of the transgender community is connected to the House Ball scene. The House Ball community, comprised of primarily black and Latino LGBT people, is organized around ball events. At Balls, groups known as "houses" compete in different event categories such as fashion and performative dance. For some transgender women, joining a House is a way of finding community and participating in a kinship network. It is also a place to find identity affirmation, as transgender and 
gender-nonconforming identities are celebrated in the House Ball scene. ${ }^{27}$ Despite these positive aspects of the House Ball community for transgender women, aspects of the Ball culture were seen by participants as barriers to engagement and retention in HIV care. For example, participants reported that the House Ball culture may reinforce substance abuse:

When you're in the ball house scene, at times it's glorified, glamorized, you know? It's like, "Oh, she's full," or, "She's high," and it has become, like, the "in" thing. To be one of the "it" girls you had to be stoned out of your mind.

Moreover, the House Ball scene was described as accepting of and perpetuating sex work, and as discussed above, transgender women who participate in sex work were thought to face greater obstacles to HIV care. HIV-negative women who join the House Ball scene and start or continue sex work may be at significant risk of seroconversion. One participant, who identified as a transgender woman and previously had been part of the House Ball community explained:

The [House family leader] that was my "mother," she was, like, an older trans woman who became my mother. She said, "Okay, well, this will be your first one. He'll give you \$50 and take you home." I looked at that man, I said ... I didn't say it but she wouldn't take no for an answer so I had to wait until she walked away and just tell him, "I'm not doing this. You need to, um, I'm not staying here. I'm not doing this." And he said, "Oh, that's alright, I'll just take you home." I was like, "I don't need you. ..." You know what I'm saying? I'm from [name of state]. I knew how to get where I needed to go and I didn't need his $\$ 50$ and I wasn't having sex with him. You know? But she wouldn't, I couldn't even tell her that. She was so adamant about, "This is what the process is." And, um, because that's what she had learned, that's what she had survived.

The House Ball scene was also seen as presenting a barrier to engagement and retention in HIV care by being insular and having a unique system of values. On the surface, the community affirms and celebrates queer and gender-nonconforming identities, but on a deeper level, it was seen as promoting unhealthy behavior, reinforcing harmful standards of beauty, and further isolating its members from the general public:

Participant: [In the House Ball community] they glamorize the sex work. They go into the balls and they're wearing the most beautiful gowns, the designers, you know? Top designers. So now you have the younger ones saying, "Well, do I do like [name] and get a job or do I do like this girl and ... be glamorous ... be a star just in this setting.

Another participant substantiated this claim by stating:

Participant: The Ball House community right now is a wreck. It started out as a family-based thing and yeah, some of the families there are still the same, but because of the competition these girls, again, over-inject silicone. I mean, overuse the silicone, overuse the hormones. Facial surgeries that, oh, my gosh, more than Joan Rivers. You know?

Interviewer: [Laughing]

Participant: I'm serious and they're only 23.

Interviewer: I think I'm getting it now, what you're saying. So in other words, it's like the Ball community is almost like medicating the pain and not going to the root.

Participant: Exactly.

Community perceptions about HIV. Many participants discussed barriers to HIV care that were related to the transgender community itself. In large urban areas like New York City, transgender women may seek out other transgender women, and the communities they form can have practices, beliefs, social norms, and internal conflicts that could hinder their members' engagement and retention in care.

One participant described a mindset in the transgender community that HIV is now seen as "just another chronic disease," even though being HIV positive remains stigmatized. This change in perception may be a barrier to care if transgender women are not treating HIV as a serious health problem warranting treatment. At the same time, if HIV is thought of as being "just like diabetes," HIV stigma may become less severe and function as less of an impediment to care. As one participant stated:

The community is beginning to see HIV as simply just another chronic problem. It's just like diabetes. Well, we know it's not.... The bad thing is that we may see diminishment of funding. The good thing is that it's easier to sell the clients on care. If it's just like diabetes, then it's not a big deal...that attitude begins to destigmatize the, um, the carrying of an HIV virus... and so it's really not a big deal anymore. Now, we know it is because we just did a memorial service on Monday for one of our clients, right? We know people are still dying from this. We know it's still killing people. We know that it's awful. So that's the bad part. We know how bad it is and they're thinking it's not so bad. But on the other hand, they're not thinking it's so bad so they...the stigma is beginning to dissolve.

Family-level barriers. Participants described barriers to care arising from relationships that transgender women have with their families, both while growing up and once they are adults.

Family support. A common trend in the narratives was the lack of family support, attributed to either outward rejection of transgender identity or to an unstable family situation before disclosure or coming out, as in the example of transgender women raised in foster care or group homes. Lack of family support was perceived as a barrier to engagement and retention in care for many reasons, including a lack of financial resources, and lack of social and emotional support:

Can you imagine? Because she was trans. Because she was beaten [by family] and she ran away and she was beaten and she ran away and she was beaten and she ran away because they can beat that out of you and that cures you, right? Of course, it doesn't because you don't cure being left-handed or blue-eyed either.

According to participants, the lack of family support contributed to housing instability, substance abuse, and mental health issues that may serve as additional barriers to care among transgender women living with HIV. Rejection by family was often related to religious dogma, as one participant related as follows:

"I told you so, I told you so. Look what happened to you. See? God didn't want you like this." And when you ... especially for a lot of these people who grow up in the church, when your parents try, attempt to take your faith away from you, tell you that God doesn't love you, God doesn't care for you, that takes another toll on a person mentally.

Another example of rejection noted by a number of participants was families' insistence that transgender women use 
their male birth names and present in the male role to be allowed to stay at home. Transgender women whose families took this approach had "support" as long as they hid their identities. Shaming and suppression of one's authentic self was noted as being a barrier to HIV care:

Participant: The couple girls I saw that went back home to families and one was really close to me. It broke my heart because she was, like, in her room and they cut off her hair. She had to wear masculine clothes. She was sick. They had the Bible and chapels and candles and all kinds of stuff around her trying to exorcise the demon of AIDS ... because they don't even know about HIV a lot of times. It's just straight AIDS. And it just ... it can break a person down, you know? If you don't have positive reinforcement around you when you are going through this difficult time, you know? It's like I think it just sucks the life out of an individual.

Interviewer: Mm-hmm.

Participant: I saw how literally the life just, like, literally drained from her body and, you know, she was such a vibrant person. Within, what, it was like six or seven months she just fell apart.

Interviewer: And then what happened?

Participant: She died. When she died, they buried her in a suit like they do with most transgender people and they block your friends from coming to see you. Closed casket funerals, closed funerals.

Family violence. Some participants described the experiences of transgender women to include a history of abuse, violence, and abandonment. Especially for young people, rejection and violence by family was viewed as robbing them of the only support system they have, often leaving them without money or shelter:

So imagine what the young person who is going through all of this being bounced around from the house where she's abused and not really understanding it's abuse, being fondled, you know?... and not to have your family support you in all that, who's been your support mechanism, you know, for the longest. I think it's really jarring and it's traumatic and I don't think people understand the trauma of just ... what transition tends to look like especially if you're poor, especially if you're undereducated, especially ... you know what I'm saying? In a violent environment, you know?

Individual-level barriers. Participants described a number of individual-level barriers to engagement and retention in HIV care based on their experience in working with transgender women living with HIV. These included competing priorities, participation in sex work, limited health literacy, and the quest to affirm gender identity.

Competing priorities. Participants explained that life stressors other than HIV often took priority for the transgender women with whom they worked. Fighting to secure basic needs, such as housing and income, often took precedence:

I think that the community is plagued because a lot of them are just trying to survive in different ways.

She's, uh, living in an apartment with some guy who just wants to use her sexually.... She's not stable enough to seek the medical care or to have someplace to have her medicines and all that. She's not even thinking ... that's, like, steps removed from where she is. She basically just doesn't even have a place on a daily basis to live.
Sex work. Two barriers to engagement and retention in HIV care emerged related to sex work. First, participants explained that the erratic schedule of sex work makes it difficult for transgender sex workers living with HIV to take their ARV medications consistently. Second, the stigma attached to being HIV-positive in the context of sex work required secrecy to preclude jeopardizing their source of income. Thus, sex work interfered with HIV care and HIV care interfered with sex work:

The lack of schedules, right? It's an up anytime, whenever the phone rings. So if you're supposed to take your medication at $2 \mathrm{PM}$, and you're in with a client and he's until $4 \mathrm{PM}$, you miss that window. Then you may not even do it at 4 PM, you may do it at 6 PM.

Well, one of the things about our clients is that they make a little extra money on the side through sex work, and if their date comes in and sees a couple of bottles of pills, "Well, what's that for?" Typically the dates already know what this is for, but they don't want to have that around because the dates might notice it and see and the dates are paying extra for sex without, uh, condoms so they don't want to be on that. They don't want it anywhere nearby because that's gonna reduce their income.

Effort required to engage in HIV care. A number of participants commented that many people living with HIV, not only transgender women, feel that the effort of engaging in care is in itself a burden. Successful engagement in care is time consuming, particularly when initiating ART. Participants noted the need for frequent visits to multiple doctors who may not be in the same location, making it hard for clients to attend all the required appointments. They also mentioned that for some clients, the need to take multiple pills at particular schedules is confusing, time consuming, and inconvenient, even for a person with stable housing and a job during the day. Additional perceived barriers related to ART itself include potential side effect and the investment of significant time and effort without an immediate benefit that can be seen or felt:

Participant: I mean, I just think that there's immediate results from hormones and things like that but there's not... they don't see any visible change from, uh, like, HIV treatment. Interviewer: Mm-hmm.

Participant: So I think that that's where the lag is. I think they want desperately to be a woman and so they're willing to do whatever they have to do, even black market stuff, even silicone injections, whatever, to get immediate results but they don't see the same, um...

Interviewer: Well, you're saying that that's a higher priority plus there's no immediate benefits they can feel in their dayto-day life.

Participant: Right.

Health literacy. Participants also identified denial and low health literacy as barriers to receiving quality HIV care. Low health literacy, especially for clients with limited education, was seen as contributing to misinformation about HIV: "There are people out there still with the idea that if you touch an HIV person you can become infected. It's like ... it's really crazy." Participants further believed that denial is a relatively common response to receiving a positive HIV diagnosis, which they understood in the context of the additional stress of such a diagnosis on top of stress related to economic instability, homelessness, or drug use: 
In a lot of cases, the clients [transgender women] are still in, uh, in deep denial about HIV, don't want to talk about it, don't want to think about it. Um, they really don't want to talk to physicians or anybody about it and they are asymptomatic.

A number of participants noted that mental health and substance use issues may further impair health literacy and health behavior, impeding transgender women's ability to make educated decisions about their lives:

I have a trans girl, I don't know if I said this, but she ... you know, you have these ADHD and these different diagnoses and then she's been in rehab since she was 13 for alcohol abuse ... and, um, who walked out into the middle of the street drunk and ... plus they're having sex just to have a place to go, right? And you can have sex with 10 men, disgusting men doing things that they couldn't get for free. Like, if they could get someone to do it for free, they would. But they're paying for sex and being treated, you know, horribly and so they're medicating to try to numb themselves so they can do these things.

Affirmation of gender identity. Not all of the individuallevel barriers to engagement and retention in HIV care described above are unique to transgender women living with HIV. The most salient individual-level, transgender-specific barrier that emerged is related to the quest to affirm gender identity. One participant described a patient recounting how proud she was when a doctor did not recognize she was transgender; her desire to pass was so strong that she did not disclose her transgender identity or her HIV status to this provider, making it difficult for the doctor to provide her with quality care. The importance of affirming gender identity may lead some transgender women to engage in high-risk behaviors to seek validation of their gender identity from others, including through assuming a traditional female gender role in sexual encounters and relationships:

Some trans women, not all, have an inclination ... to use sex to validate their realness, their passability. So now they're going out there with guys and from the time a guy looks at them they say, "Oh, wow, he's straight, I believe he's straight, so that means that I'm real so I'm gonna sleep with him." And if he says he doesn't want to use condoms, they go right ahead with it.

The need to affirm gender identity may also lead to unsafe injection practices of hormones and/or silicone:

They tend to, uh, buy the hormones [on the] black market. Um, they take silicone injections from people in hotel rooms and stuff like this. The silicone injections are not safe because number one, they don't know what they're putting into their bodies, and number two, sometimes they don't change the needles, even though people stress that. So you may have, uh, a lot of infections from HIV ... you may have a lot of infections from needle sharing.

Finally, gender affirmation through hormone therapy or surgery often takes precedence over HIV care. For many transgender women, these medical interventions are regarded as essential to align their body with their gender identity. Participants mentioned that gender dysphoria can be so severe that it becomes the dominant concern in transgender women's lives:

Well, you know, to begin with most of the clients that show up here because there are two things that they want. Uh, they both start with "h." They want housing and they want hormones.
Um, our clients, um, in some cases they'll come with very low CD4 count and the physician will say, "Let's get you healthy before I let you start on hormones." And that's not what anybody wants to hear, you know, if you're 18 and want it now.

Moreover, ARV medications are perceived by some in the transgender community as interfering with the desired benefits of feminizing hormone therapy. This may be related to lipodystrophy, a potentially unsightly remodeling of the body's fat stores, which has been a well-known side effect of some ARV medications. For transgender women who seek to attain femininity and beauty to affirm gender identity, such potential side effects were seen as detrimental:

I mean, honestly, a lot of trans women have... women in general have body image issues. Um, I've been in and out of all kinds of groups for the last God knows how many years. They have body issues, body image issues. Trans women have body image issues. Uh, and if the ARVs are going to mess with my body image which is already messed up, you know, I'm starting out with a bad body image and if this is going to "f" it up a little bit, well...

\section{Strategies to mitigate barriers to engagement and retention in HIV care}

Participants identified several key facilitators of successful HIV care for transgender women. Paramount among these facilitators was a strong client/provider relationship, defined by effective communication, compassion, and active listening, as well as a foundational knowledge of transgender issues. Participants felt strongly that professionals need to be prepared to address health concerns related to transgender identity, including medical knowledge to provide highquality primary health care and gender-affirming health care in addition to HIV care tailored to the needs of transgender women. Participants also urged providers to gain comfort, understanding, and skills in treating people who are transgender by incorporating transgender-specific cultural and clinical competence in their training.

For example, participants described the importance of communicating genuine caring in their interactions with transgender patients as follows:

So you go into your doctor's office. He says, "Hi, how are you?" And he continues to do your work. [A particular doctor], when you go in to him, it's more of a personal conversation ... Where you feel engaged. So, "What did you do this week?" You know? Not just, "Put your arm out, let me take your blood." And then explain to these girls what the medications are that they're taking and explain to them ... really, really, like, listen. It's not like you just hand the girl a prescription.

The need to use appropriate language and terminology in practice was seen as paramount, including the use of preferred names and pronouns (i.e., based on gender identity rather than sex assigned at birth) and the development and use of forms and computer systems that reflect their identities and needs (e.g., providing gender-diverse options on intake forms and paperwork). They also emphasized the importance of patient education to improve health literacy and to empower transgender women to advocate for themselves in health and social service settings. 
Exemplifying the call for providers who understand their clients' identities, experiences, and needs, some participants noted the value of transgender-identified health and social service providers who reflect the client's gender identity:

Only I can say that as a staff person who's trans, you know? Somebody else can't say, "Oh, you should be okay with being trans," because it doesn't mean the same thing. Or, "Being trans is fine." But it's different when it's staff saying it, you know? I mean, when it's a person who's trans saying it.

The importance participants placed on personal connection to and reflection of the transgender community is evident in one participant's suggestion for a trans woman spokesperson living with HIV, a visible member of the transgender community advocating for the benefits of engagement and retention in HIV care. Within the context of working with the House Ball community, such a champion was proposed to facilitate consistent engagement in HIV care by modeling self-empowerment related to health and wellbeing:

His name is ___ and, um, he's on some of the campaigns and when people saw those campaigns they participated in the survey more because there was someone who was just like them that was not afraid to say, "I am HIV-positive, I'm taking my meds, I'm doing this." And when you have someone brave enough to stand out there and say, "Look, I'm doing this because I care about you," I think you would get some of them to become very much engaged.

Many of the suggestions made by participants to break down barriers to HIV care reflected the need for integrated care management. Participants underscored the importance of interdisciplinary collaboration among providers caring for transgender women living with HIV and the provision of multiple services under one roof-including HIV care, transgender health care, mental health care, substance abuse treatment, and social services:

I believe that if hormone therapy was directly linked with mental care, once you start hormones, you start mental care, I think that will set up for the individual to become more comfortable with their practitioner, for the individual to understand what they're going through and what the terms are that they're using, explain to them the pros and cons of the transition.

Participants expressed the need for safety and security, including confidential and accessible health and support services. For example, participants argued that the hours of operation of these services should respond to the safety and accessibility concerns expressed by their clients. One participant summed it up as follows:

I think it should be someplace that is accessible to them, easy to get to and not scary to get to, and, um, I think it should just be another thing that they do. Like, if they have, uh, mental health issues, HIV or transition, just all that care would be taken care of in a place that's kind of ... I don't want to say obscure but, like, you wouldn't be able to know all of that's going on.

Other ideas to facilitate engagement and retention in HIV care were community engagement programs and smartphone applications to promote ARV medication adherence. Finally, to facilitate successful referrals, participants suggested patient navigation services to escort clients to appointments, particularly for newly diagnosed transgender women.

\section{Discussion}

Engaging and retaining transgender women living with HIV in consistent HIV care is crucial for improving health and longevity in this population, as well as limiting further HIV transmission by individuals with high viral loads. Health care and social service providers are working to stem the HIV/AIDS epidemic in New York City, the city with the greatest burden of HIV in the United States. Their insights provided a unique and valuable perspective on the challenges encountered in providing compassionate and high-quality HIV care to transgender women. However, it is important to note that this study focused on the needs of a small subset of the transgender community, specifically transgender women living with HIV, and that findings should not be interpreted as reflecting the entire, very diverse population of transgender women.

Stigma was an overarching factor at every level of barriers and facilitators to care. Often, participants' perspectives reflected intersections of stigma and other factors at play. For example, at the health systems level, reports of disparaging treatment of transgender women by clinic staff could be explained as individual bias, a lack of cultural competency training, or a gross oversight at the administrative level to address transgender issues in its care delivery model.

Similarly, stigma was found to be operating as internalized stigma attached to being transgender or HIV positive; as enacted and felt stigma within the House Ball community between transwomen that engaged in sex work and those that did not; as enacted stigma in the general population toward transgender women, people living with HIV, racial/ethnic minorities, and the economically disadvantaged; and within the health care system, as a lack of cultural competence or outright refusal to treat transgender women.

Internalized stigma was an important factor that prevented transgender women from seeking care. Fear of enacted stigma was omnipresent. The intersectionality of multiple stigmatized identities related to transgender identity, HIV status, race/ethnicity, socioeconomic status, and sex work compounded the challenges faced by transgender women engaging in HIV care. In addition, mental health, substance use, and housing instability were identified as common barriers to care, each of which carry additional stigma. Further research is needed to better understand how transgender women cope with these multiple stigmas and how their negative impact on engagement and retention in HIV care can be mitigated.

All study participants emphasized the importance of cultural and clinical competence in transgender health in reducing the identified barriers to engagement and retention in HIV care. These competencies include expert knowledge on the part of medical providers, professional respect of providers and office staff, and the use of preferred names and pronouns on intake forms and in information systems. The responsibility for creating inclusive and safe places to deliver care falls on providers and their institutions.

Interventions to improve engagement and retention of transgender women in HIV care must be multifaceted, addressing various areas of health and wellbeing (e.g., HIV, physical health, gender affirmation, mental health, substance use, housing), and be ongoing so they can permeate the culture of service institutions. At the administrative level, a diverse staff inclusive of the perspectives of transgender 
women is an asset, and health and information systems should reflect the diversity in gender identity, gender expression, and sexual orientation found within the transgender community. Sensitivity training to equip staff with information and skills to serve transgender women with HIV is urgently needed.

At the individual level, providers must forge strong provider/patient relationships, overcoming mistrust many transgender women have because of previous negative experiences in interactions with health and social service providers.

Nearly every participant in this study described a similar vision for the ideal provision of care to transgender women living with HIV. This vision is that of comprehensive, integrated care. Many such comprehensive, integrated care practices exist in New York, such as Patient-Centered Medical Homes, yet few of these practices are prepared to competently care for transgender women.

Health policy should focus on resources to strengthen existing patient-centered medical homes, especially those for people living with HIV, and the creation of new ones accessible and equipped to provide culturally and clinically competent care. Within these integrated care centers, a variety of services able to meet as many of the patient's needs as possible should be available, such as gender-affirming care (counseling, hormones, surgery, access to peer support), HIV care and prevention (testing, measuring viral load, monitoring adherence to antiretroviral medications, checking for side effects, and counseling with regard to disclosure, condom/barrier use, and PrEP for partners), primary care (general wellness visits, sick visits, sexually transmitted infections (STI) testing, prostate exams, and other preventive care), health education and wellness programming (patient education with regard to nutrition, exercise, advocacy), mental health care, case management, and social work support, including housing.

Systems must be nurtured to promote interprofessional collaboration and coordination among an interdisciplinary cadre of health and social service providers. An inclusive, accessible, safe, confidential, competent, and efficient integrated care model should be the gold standard for optimizing engagement and retention of transgender women in HIV and overall health care. Moreover, such integrated care could be provided to all patients, regardless of their gender identity or HIV status (i.e., a HIV status-neutral care site), further alleviating transgender women's concerns about privacy and confidentiality.

Finally, within the context of integrated care or not, based on the perspectives shared by health and social service providers in our study, access to HIV care in evening and nighttime hours, outreach efforts that may include a spokesperson to promote engagement and retention in care, the expression of genuine understanding and caring, and efforts to promote self-efficacy and empowerment are recommended.

Through our qualitative approach, we attempted to obtain a range of perspectives rather than trying to estimate how often which type of barriers occur and for whom; the insights gained may generate new hypotheses for future quantitative research or inform the development and subsequent testing of tailored intervention strategies. However, our study was limited by the small sample size $(N=19)$, although data saturation was achieved when we no longer uncovered novel themes regarding barriers and facilitators to HIV care, which has been shown to occur after as few as 12 interviews. $^{28}$
Participants were recruited in the metropolitan New York City area, which limits generalizability of findings to other cities, rural areas, and other countries. Also, although the inclusion of transgender-identified health and social service providers added valuable insights to understanding barriers and facilitators to engagement and retention in HIV care, it is important to note that their responses to our questions likely also reflected their personal experiences as community members and clients, in addition to their experiences as health and social service providers.

Future research should examine the intricacies of engagement and retention in care among transgender women living with HIV from various points of view, including from the patients themselves, to further shed light on how barriers can be reduced and engagement and retention can be facilitated. Further research should also evaluate the impact of the aforementioned recommendations once they have been implemented.

Ongoing research must examine the changing landscape of health care, especially as it affects such vulnerable populations as transgender women living with HIV in the context of the current sociopolitical climate. Finally, research is also needed to determine the barriers and facilitators to HIV testing and PrEP use among HIV-negative transgender women. Some of the findings from our study focused on engagement and retention in care for transgender women living with HIV may also benefit the uptake of PrEP among the larger population of transgender women and their partners at risk for HIV infection and transmission.

\section{Author Disclosure Statement}

No competing financial interests exist.

\section{Funding Information}

The research on which this report is based was funded by grants from the National Institutes of Health (P30-MH43520, Robert H. Remien, PI) and The MAC AIDS Fund (MAF CU13-3233, Walter Bockting, PI).

\section{References}

1. Centers for Disease Control and Prevention. HIV and AIDS data through December 2015 provided for the Ryan White HIV/AIDS Program, for fiscal year 2017. HIV Surveill Suppl Rep 2017;22. Available at: www.cdc.gov/hiv/library/ reports/hiv-surveillance.html (Last accessed November 2017).

2. New York City Department of Health and Mental Hygiene. HIV Surveillance Annual Report, 2015. 2017. Available at: www1.nyc.gov/assets/doh/downloads/pdf/dires/hiv-surveillanceannualreport-2015.pdf (Last accessed December 2, 2019).

3. White House Office of National AIDS Policy. National HIV/AIDS Strategy for the United States. 2010. Available at: https://www.whitehouse.gov/sites/default/files/uploads/ NHAS.pdf (Last accessed December 2, 2019).

4. New York State Department of Health. 2015 Blueprint for achieving the goal set forth by Governor Cuomo to end the epidemic in New York State by the end of 2020. 2015. Available at: https://www.health.ny.gov/diseases/aids/ending the_epidemic/docs/blueprint.pdf (Last accessed December 2, 2019).

5. Castilla J, del Romero J, Hernando V, et al. Effectiveness of highly active antiretroviral therapy in reducing heterosexual transmission of HIV. J Acquir Immune Defic Syndr 2005;40:96-101. 
6. Cohen MS, Chen YQ, McCauley M, et al. Prevention of HIV-1 infection with early antiretroviral therapy. N Engl J Med 2011;365:493-505.

7. Cohen MS, Chen YQ, McCauley M, et al. Antiretroviral therapy for the prevention of HIV-1 transmission. N Engl J Med 2016;375:830-839.

8. Das M, Chu PL, Santos G-M, et al. Decreases in community viral load are accompanied by reductions in new HIV infections in San Francisco. PLoS One 2010;5:e11068.

9. Robertson M, Larague F, Mavoricolas H, et al. Linkage and retention in care and the time to HIV viral suppression and viral rebound-New York City. AIDS Care 2015;27:260267.

10. Mannheimer SB, Matts J, Telzak E, et al. Quality of life in HIV-infected individuals receiving antiretroviral therapy is related to adherence. AIDS Care 2005;17:10-22.

11. Herbst J, Jacobs E, Finlayson T, et al. Estimating HIV prevalence and risk behaviors of transgender persons in the United States: A systematic review. AIDS Behav 2007;12: $1-17$.

12. Reisner SL, Jadwin-Cakmak L, Sava L, et al. Situated vulnerabilities, sexual risk, and sexually transmitted infections' diagnoses in a sample of transgender youth in the United States. AIDS Patient Care STDS 2019;33:120-130.

13. Baral S, Poteat T, Stromdahl S, et al. Worldwide burden of HIV in transgender women: A systematic review and metaanalysis. Lancet Infect Dis 2013;13:214-222.

14. Melendez RM, Exner TA, Ehrhardt AA, et al. Health and health care among male-to-female transgender persons who are HIV positive. Am J Public Health 2006;96:1034-1037.

15. Sevelius JM, Carrico A, Johnson MO. Antiretroviral therapy adherence among transgender women living with HIV. J Assoc Nurses AIDS Care 2010;21:256-264.

16. Deutsch MB, Glidden DV, Sevelius J, et al. HIV preexposure prophylaxis in transgender women: A subgroup analysis of the iPrEx trial. Lancet 2015;2:e512-e519.

17. Bockting W, Robinson B, Forberg JS. Evaluation of a sexual health approach to reducing HIV/STD risk in the transgender community. AIDS Care 2005;17:289-303.

18. Garofalo R, Johnson AK, Kulns LM, et al. Life Skills: Evaluation of a theory-driven behavioral HIV prevention intervention for young transgender women. J Urban Health 2012;89:419-431.
19. Nemoto T, Operario D, Keatley J, et al. Promoting health for transgender women: Transgender Resources and Neighborhood Space (TRANS) Program in San Francisco. Am J Public Health 2005;95:382-384.

20. Taylor RD, Bimbi DS, Joseph HA, et al. Girlfriends: Evaluation of an HIV-risk reduction intervention for adult transgender women. AIDS Educ Prev 2011;23:469-478.

21. Remien R, Bauman LJ, Mantell JE, et al. Barriers and facilitators to engagement of vulnerable populations in HIV primary care in New York City. J Acquir Immune Defic Syndr 2015;69(Suppl 1):S16-S24.

22. Sevelius JM, Patouhas E, Keatley J, et al. Barriers and facilitators to engagement and retention in care among transgender women living with human immunodeficiency virus. Ann Behav Med 2014;47:5-16.

23. Brofenbrenner U. Toward an experimental ecology of human development. Am Psychol 1997;32:513-531.

24. Brofenbrenner U. The Ecology of Human Development. Cambridge, MA: Harvard University Press; 1979.

25. MeLeroy KR, Bibeau D, Steckler A, et al. Ecological perspective on health promotion programs. Health Educ Q 1988;15:351-377.

26. Hsieh HF, Shannon SE. Three approaches to qualitative content analysis. Qual Health Res 2005;15:1277-1288.

27. Colon ER. Getting Life in Two Worlds: Power and Prevention in the New York City House Ball Community. Dissertation, Doctorate of Philosophy, Rutgers University; 2009.

28. Guest G, Bunce A, Johnson L. How many interviews are enough? An experiment with data saturation and variability. Field Methods 2006;18:59-82.

Address correspondence to: Walter Bockting, PhD

HIV Center for Clinical and Behavioral Studies Division of Gender, Sexuality, and Health New York State Psychiatric Institute Columbia University Irving Medical Center 1051 Riverside Drive Unit 15

New York, NY 10032

E-mail:wb2273@cumc.columbia.edu 


\section{Appendix A: Interview Guide}

\section{Introduction}

We are interviewing providers of HIV care about HIV+ patients and the problems they experience in staying in care. As someone with experience treating people with HIV, we are asking for your help in understanding why some patients are "linked" to HIV care-they actually do see a providerbut drop out of care. We are working with the NYC Department of Health and Mental Hygiene on this project, and we plan to identify strategies for helping patients stay engaged in care so they can experience the benefit of treatment.

\section{Background}

1. Why do you think it is important for HIV+ patients to stay consistently engaged in HIV care?

a. PROBE: How soon do you think HIV care should begin?

b. PROBE: How soon should patients begin ARVs?

2. In your experience, are there many HIV+ patients who are not receiving care for their HIV?

a. PROBE: How big a problem is this for transgender women?

3. What do you think that HIV+ patients understand about engaging and staying in HIV care?

a. PROBE: Timing of entering into care

b. PROBE: Effects of inconsistent care on health

\section{Challenges}

4. Why is it difficult for HIV+ transgender women to engage and stay in care?

a. PROBE: Can you tell me about health system barriers and give me some specific examples?

i. FURTHER PROBE IF NECESSARY: Other people we have talked to have mentioned costs of care, policies, access to care (e.g., location of health center/clinic; hours of services; long waiting time; services are not patient-centered; mistrust of health care system or providers)?

b. PROBE: Can you tell me about community barriers and give me some specific examples?

i. FURTHER PROBE IF NECESSARY: Other people we have talked to have mentioned stigma as a community barrier.

c. PROBE: Can you tell me about family barriers and give me some specific examples?

i. FURTHER PROBE IF NECESSARY: Other people we have talked to have mentioned nondisclosure of HIV+ status; lack of family support.

d. PROBE: Can you tell me about barriers that individuals face and give me some specific examples?

i. FURTHER PROBE IF NECESSARY: Other people we have talked to have mentioned substance use; depression or psychological stress; skepticism about the benefits of HIV treatment; fear of being stigmatized; not feeling ill; treatment fatigue; pill burden.

\section{Solutions}

5. What things can we do to help keep HIV+ transgender women engaged in HIV care?

a. PROBE: What do you think we can do to help transgender women stay in care while they are still engaged?

b. PROBE: Support groups, patient navigators, transportation?

6. Do you know of other programs or initiatives focused on helping these patients to stay engaged in care?

a. PROBE: What approaches have been tried that worked? Can you give me some specific examples?

b. PROBE: What approaches have been tried that did not work? Can you give me some specific examples?

7. If you could do things differently to keep HIV+ transgender women engaged in care, how would you do it?

a. Imagine that you were given an unlimited amount of money to help transgender women engage and stay in HIV care. What things would you change to increase this population's initiation and retention in HIV care? What new strategies would you introduce to get people to stay in care?

b. Can you tell me about a situation you recently experienced that helped an $\mathrm{HIV}+$ transgender woman stay in care?

c. Is there anything else you think I should know?

8. Have you heard about the concept of 'treatment as prevention'? What have you heard?

a. Some studies have shown that when people receive treatment for HIV they are less likely to transmit it to others. What do you think about the concept of 'treatment as prevention' 